\title{
Dry eye and Meige's syndrome
}

Kazuo Tsubota, Tsutomu Fujihara, Minako Kaido, Asako Mori, Masaru Mimura, Motoichiro Kato

\begin{abstract}
Aims-To determine the relation between dry eye and Meige's syndrome.

Methods-325 patients with dry eye were divided into those responsive to topical and other forms of treatment $(n=276)$ and those who were not $(n=49)$. A neuropsychiatric examination was performed to check for Meige's syndrome in the latter group.

Results-Twenty eight (57\%) of the treatment unresponsive patients were diagnosed with Meige's syndrome.

Conclusions-There is a subgroup of patients with dry eye who do not respond to simple therapy. More than half of these patients have Meige's syndrome and need psychiatric, as well as ophthalmic, care. (Br f Ophthalmol 1997;81:439-442)
\end{abstract}

Dry eye syndrome is an ocular surface disorder associated with tear film abnormalities and potentially significant discomfort caused by burning or foreign body sensation, chronic injected eye, increased blinking, or ocular fatigue. ${ }^{12}$ Dry eye associated with Sjögren's syndrome is the most prominent form of the condition, but dry eye is the subject of active research and other forms have now been identified. $^{3}$ Treatment for dry eye is for symptomatic relief and is directed at preventing ocular surface desiccation. ${ }^{4}$ Approaches include using lubricant eye drops, wearing protective eye glasses, and performing punctal occlusion. ${ }^{4-6}$ When blepharitis or meibomianitis is present, lid hygiene with antiinflammatory drugs are effective. ${ }^{3}$ Most patients achieve at least partial relief, although long term treatment - often with a combination of techniques - is usually required. However, some patients with dry eye do not respond to any treatment, and may complain that they cannot keep their eyes open.

Blepharospasm is another condition in which patients cannot keep their eyes open..$^{7-10}$ It was recognised in the 19 th century and was first reported in 1910 by Meige as bilateral facial convulsion or dystonia, characterised by intermittent or persistent involuntary bilateral eye closure caused by spasmodic contractions of the orbicularis oculi muscles. ${ }^{8}$ Since blepharospasm is often associated with spasms of the middle and lower facial or oromandibular muscles, the condition is considered a 'blepharospasm-oromandibular dystonia' and is often referred to as Meige's syndrome. ${ }^{10-12} \mathrm{It}$ frequently interferes with such activities of daily living as watching TV, driving, and read- ing. In mild cases, Meige's syndrome occurs only with certain stimuli such as exposure to bright light, wind, or air pollution. ${ }^{\circ}$ As described by Meige, spasms tend to be triggered when patients look up and inhibited when they close their eyes or rest in a dark room. ${ }^{8}$ We have previously shown that looking up is accompanied by increased ocular surface area, which increases desiccation and hence may cause or exacerbate dry eye. ${ }^{13}{ }^{14}$ Conversely, eye closure prevents desiccation of the ocular surface. Holds et al also pointed out that dry eyes frequently exacerbate essential blepharospasm and that treatment of the former may alleviate the latter, ${ }^{10}$ so that the two conditions may be at least partially related. In order to study this relation, we referred the patients with dry eye who did not respond to local dry eye therapy to a psychiatrist (MK) and a neurologist (MM), to determine whether refractory dry eye patients may actually be suffering from Meige's syndrome.

\section{Materials and methods}

DRY EYE PATIENTS

Dry eye was diagnosed in the presence of both compromised tear dynamics and ocular surface abnormalities as previously reported. ${ }^{41} \mathrm{~Pa}-$ tients who were suspected of having dry eye on the basis of such complaints as ocular irritation, fatigue, or persistent injection underwent objective examinations. If the patients had both abnormal tear dynamics as determined by the Schirmer test (less than $10 \mathrm{~mm}$ ), cotton thread test (less than $20 \mathrm{~mm}$ ), or tear break up time (BUT) (less than 5 seconds), and positive vital staining of the ocular surface by rose bengal or fluorescein, they were considered to have dry eye. ${ }^{1{ }^{15-17}}$ We also diagnosed dry eye in patients with only decreased BUT and no vital staining of the ocular surface, ${ }^{18}$ because the severity of their complaints warranted inclusion in our study.

From July 1992 until June 1994, 346 new patients in the department of ophthalmology, Tokyo Dental College, Ichikawa General Hospital, satisfied the criteria for traditional dry eye and dry eye with only decreased BUT. Throughout the study, we examined the worse eye from each patient, as determined by the simple Schirmer test.

\section{TEAR EXAMINATION}

BUT was measured with $1 \mu \mathrm{l}$ of $1 \%$ fluorescein solution without anaesthesia or preservatives. Three measurements were taken and then averaged.

The Schirmer value with anaesthesia and the tear clearance rate (TCR) were measured 5 minutes after instilling a $10 \mu \mathrm{l}$ drop of $0.5 \%$
Accepted for publication 13 January 1997 
fluorescein and $0.4 \%$ oxybuprocaine hydrochloride into the conjunctival sac. ${ }^{19} \mathrm{~A}$ standard Schirmer test strip was then placed for another 5 minutes. The length of the wet portion was measured and the intensity of its staining was compared with the standard strip colours for the Schirmer test with anaesthesia or TCR. The TCR was determined by the rate at which the colour of the fluorescein dye faded, and was graded $1,1 / 2,1 / 4,1 / 8,1 / 16,1 / 32,1 / 64,1 / 128$, or $1 / 256$. The Schirmer result is believed to reflect the basic and some reflex tearing whereas the TCR value reflects the ability of the tear film layer to dilute chemicals or clear foreign bodies. The tear function index (TFI) was defined as the result of the Schirmer test with anaesthesia divided by the TCR. ${ }^{19}$

The Schirmer test with nasal stimulation was originally described by Schirmer as the Schirmer II test, and was considered to measure reflex tearing. ${ }^{20}$ In our study after determining Schirmer values for 5 minutes without topical anaesthesia, we inserted a small cotton swab $(8 \mathrm{~cm}$ long, $3.5 \mathrm{~mm}$ wide at the top) into the patient's nasal cavity slightly upward and parallel to the lateral wall. We then placed the Schirmer paper in the conjunctival sac as with the regular Schirmer test, while the cotton applicator was kept in place for 5 minutes. $^{2122}$

OCULAR SURFACE EXAMINATION

Vital staining of the ocular surface was performed with a fixed concentration and volume of dye ( $2 \mu \mathrm{l}$ of $1 \%$ rose bengal mixed with $1 \%$ fluorescein). ${ }^{23}$ The staining intensity was evaluated according to a semiquantitative scale described previously, with maximum values of 9 for rose bengal and 3 for fluorescein. ${ }^{24}$

TREATMENT RESPONSIVE AND TREATMENT UNRESPONSIVE DRY EYE PATIENTS

Initially, dry eye patients wore protective glasses and frequently used preservative-free artificial tears. ${ }^{45}$ Patients who had meibomianitis used a combination of topical and oral tetracycline and were instructed in lid hygiene. The second step included the use of a mild topical steroid (Flumethoron, Santen Pharmaceutical, Osaka, Japan $0.1 \%$ fluoromethalone, four times a day) or antiallergic medication (Intal, four times a day, Fujisawa Pharmaceutical Co Ltd, Osaka, Japan or Zajiden four times

Table 1 Patient profiles of treatment responsive and treatment non-responsive dry eyes

\begin{tabular}{llll}
\hline & Responsive & Non-responsive & p Value \\
\hline Age (years) & $52.4(15.0)$ & $48.9(14.3)$ & 0.118 \\
Sex (M:F) & $43: 254$ & $24: 25$ & 0.000 \\
Tear function: & $3.9(2.8)$ & $3.4(1.9)$ & 0.631 \\
$\quad$ Break up time (s) & $6.9(6.5)$ & $8.9(8.2)$ & 0.045 \\
Schirmer-I test (mm) & $16.7(18.1)$ & $14.1(11.5)$ & 0.823 \\
Tear clearance (X) & $114.3(224.8)$ & $110.1(138.8)$ & 0.121 \\
$\quad$ Tear function index & $18.6(14.2)$ & $26.6(15.9)$ & 0.007 \\
$\quad$ Nasal stimulation (mm) & $2.8(2.8)$ & $1.2(1.2)$ & 0.000 \\
Ocular surface conditions: & $2.2(2.3)$ & $1.3(1.4)$ & 0.018 \\
$\quad$ Rose bengal score & $48 / 297$ & $1 / 49$ & 0.016 \\
Fluorescein score & $43 / 297$ & $1 / 49$ & 0.029 \\
Systemic conditions: & $44 / 297$ & $1 / 49$ & 0.026 \\
$\quad$ ANA (>160) & $11 / 297$ & $0 / 49$ & 0.353 \\
RA (>160) & & & \\
SS-A & & & \\
SS-B & & & \\
\hline
\end{tabular}

Each value shows average (SD). $p$ Value was calculated by Mann-Whitney U test or $\chi^{2}$ test. a day, Santen Pharmaceutical, Osaka, Japan). At 1 month after institution of the above treatment, 297 patients $(85.8 \%)$ reported at least some subjective improvement, while 49 patients $(14.2 \%)$ did not appreciate any improvement. The latter group of dry eye patients was considered unresponsive to local therapy.

DIAGNOSIS OF MEIGE'S SYNDROME AND BLEPHAROSPASM

Forty nine treatment unresponsive patients who had been referred for neuropsychiatric examinations were videotaped over several sessions. Meige's syndrome was diagnosed in the presence of blepharospasm and oromandibular dystonia. Careful attention was paid to prolonged spasms of eye closure, brief chronic repetitive contractions of the orbicularis oculi muscles, and dystonic involuntary movements of the facial, masticatory, labial, lingual, and mandibular muscles. Patients' medical records and histories were reviewed to exclude those exposed to neuroleptic drugs. None of these patients had any history of antipsychotic medication or neuroleptic induced parkinsonism or other involuntary movements. Neurological and neuropsychological examinations revealed no pyramidal, cerebellar, sensory, or intellectual deficits. Neuroradiological studies, in particular brain computed tomography or magnetic resonance imaging, were similarly negative.

DIAGNOSIS OF SJOGREN'S SYNDROME AND SUPERIOR LIMBIC KERATITIS

Special attention was paid to the diagnosis of Sjögren's syndrome and superior limbic keratitis. These two conditions are associated with dry eye and are considered difficult to control. ${ }^{25}$ We used the criteria of Fox et al to diagnose Sjögren's syndrome. ${ }^{26}$ We checked the serum levels of autoantibodies of SS-A, SS-B, anti-nuclear antibody (ANA), and rheumatoid factor (RA), as previously described.

\section{Results}

A profile of treatment responsive and treatment unresponsive dry eye patients is listed in Table 1 . The age difference between the two groups was not statistically significant ( $p$ $<0.05)$ whereas the difference in sex distribution-43 males (14\%) and 254 females $(85 \%)$ in the former group and 25 males $(51 \%)$ and 24 females $(49 \%)$ in the latter group-was significant. Schirmer test values were also high in the latter group as were the nasal stimulation results. Thirty five patients in the unresponsive group had less than $5 \mathrm{~mm}$ of tear break up time. Staining with either rose bengal or fluorescein and the levels of each autoantibody were significantly greater in the treatment responsive group.

Of the 49 treatment unresponsive dry eye patients, 28 (16 males and 12 females) were diagnosed with Meige's syndrome. Neither tear function nor any of the ocular surface variables differed significantly between this group and those treatment unresponsive dry eye patients found not to have Meige's syndrome (Table 2). 
Table 2 Tear function and ocular surface evaluations of Meige's and non-Meige's syndrome, and treatment responsive groups

\begin{tabular}{llll}
\hline & Meige & Non-Meige & p Value \\
\hline Age (years) & $49.7(13.5)$ & $47.9(15.6)$ & 0.693 \\
Sex (M:F) & $16: 12$ & $8: 13$ & 0.231 \\
Tear function: & & & \\
$\quad$ Break up time (s) & $3.4(1.7)$ & $3.4(2.2)$ & 0.793 \\
Schirmer-I test (mm) & $8.9(6.9)$ & $8.9(9.7)$ & 0.358 \\
Tear clearance (X) & $12.3(8.9)$ & $16.5(14.1)$ & 0.360 \\
$\quad$ Tear function index & $92.4(106.9)$ & $133.7(172.6)$ & 0.968 \\
Nasal stimulation (mm) & $28.1(16.8)$ & $23.3(14.0)$ & 0.813 \\
Ocular surface conditions: & & & \\
Rose bengal score & $0.9(1.6)$ & $1.7(1.7)$ & 0.014 \\
Fluorescein score & $1.4(1.5)$ & $1.1(1.1)$ & 0.586 \\
Systemic conditions: & $1 / 28$ & & \\
ANA (>160) & $1 / 28$ & $0 / 21$ & 1.000 \\
RA (>160) & $1 / 28$ & $0 / 21$ & 1.000 \\
SS-A & $0 / 287$ & $0 / 21$ & 1.000 \\
SS-B & &
\end{tabular}

Each value shows average (SD). p Value was calculated by Mann-Whitney U test, $\chi^{2}$, or Fisher's test.

Only one of the Meige's syndrome patients (66 year old male) proved to have Sjögren's syndrome according to the Fox criteria for autoantibodies (positive SS-A, SS-B, ANA $(>160)$, or RA $(>160))$. Furthermore, only one patient showed SS-A autoantibody in nonMeige's syndrome. Superior limbic keratitis was found in two patients with Meige's syndrome ( 32 year old male and 55 year old male) and one patient without Meige's syndrome (26 year old female).

Psychiatric examination revealed that one Meige's syndrome patient also had depression (54 year old female), while two others also suffered from alcoholism (44 year old male and 57 year old male). In the non-Meige's syndrome group, two patients were diagnosed with hemifacial spasm ( 68 year old male and 69 year old female) and two others were diagnosed with depression (75 year old male and 32 year old male).

CASE REPORT

In February 1993 a 56 year old male was referred to our dry eye clinic following poor response to ordinary dry eye therapy. The patient (Fig 1) complained of difficulty in opening his eyes especially when driving or using a video display terminal (VDT), and suffered from ocular fatigue, irritation, and occasional injection. Both eyes had Schirmer values with topical anaesthesia of $5 \mathrm{~mm}, 16 \mathrm{X}$ clearance, TFI of 80 , rose bengal scores of 2 , and fluorescein scores of 1 . The diagnosis of dry eye was confirmed by the constellation of symptoms, compromised tear function, and ocular surface staining. We increased the use of artificial tears, started the use of protective eyeglasses and tetracycline ointments, and encouraged lid hygiene for concomitant mild blepharitis.

After 1 month, the patient remained symptomatic. Topical anaesthetic (oxybuprocaine $0.4 \%$ ) provided some comfort and allowed the patient to partially open his eyes, suggesting that ocular surface factors play some role in the condition. The patient was referred to a neuropsychiatrist (MK).

Neurological examination revealed sustained forceful contraction of both orbicularis oculi muscles, associated with brief chronic

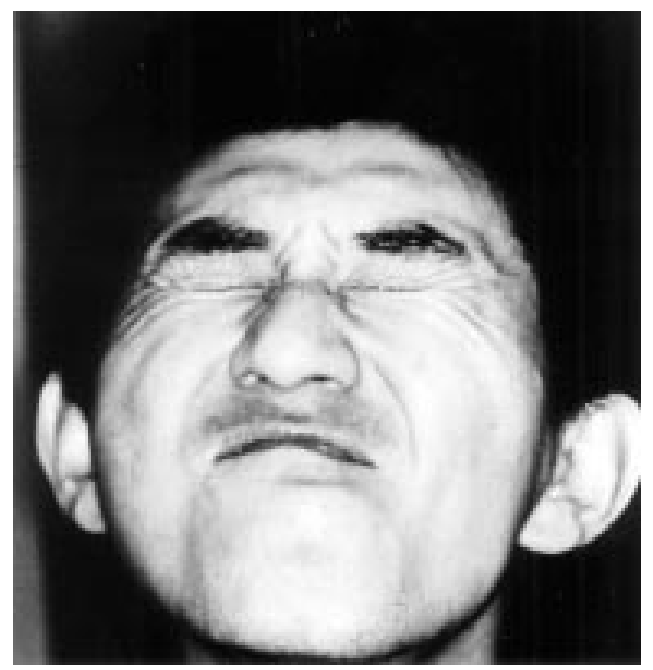

Figure 1 Typical features of Meige's syndrome. Note the blepharospasm is accompanied by mild to moderate spasms of frontalis and dystonic movements of the lower facial oromandibular muscles.

spasm. This blepharospasm was accompanied by mild to moderate spasms of the frontalis muscle and dystonic movements of the lower facial or oromandibular muscles. His dystonia was primarily on the left side of his face. No other neurological abnormalities were noted. Further questioning revealed that he sometimes became functionally blind and could not read a newspaper or enjoy television. His blepharospasm was often exacerbated by exposure to bright light or wind, and was relieved by diversions such as touching his forehead or singing. Trihexyphenidyl (up to $12 \mathrm{mg} /$ day) and clonazepam ( $4 \mathrm{mg} /$ day) reduced the severity of the contractions.

\section{Discussion}

Dry eye syndrome is a major ocular surface disorder which affects millions of people. Complaints vary from simple irritation to ocular fatigue and difficulty keeping the eyes open. ${ }^{1}$ In this study, we have shown that some dry eye patients who do not respond to local therapy such as wearing protective eyeglasses or frequently using artificial tears actually have Meige's syndrome. Although Meige's syndrome may be easily diagnosed when the chief complaint is difficulty in opening the eyes, ${ }^{8}$ atypical or mild cases exist and an appreciation of the association between dry eye and Meige's syndrome is critical if patients are to receive appropriate diagnosis and treatment.

In our clinic we perform comprehensive examination of tear dynamics and the ocular surface and have very strict criteria for diagnosing dry eye, which each of our patients satisfied. ${ }^{128}$ Although there is no perfect treatment for dry eye patients, use of artificial tears and other means of preventing desiccation provide at least some relief. In our study, 297 (85.8\%) of 346 patients reported improvement after local therapy, whereas 49 patients $(14.2 \%)$ felt no subjective relief. We have previously observed that some dry eye patients also suffer from blepharospasm, and that those patients are difficult to control with topical 
therapy alone. We thus referred the 49 treatment unresponsive patients in this study to a neuropsychiatrist, and $28(57.1 \%)$ were diagnosed with Meige's syndrome. Some sufferers of Meige's syndrome or blepharospasm do respond to standard dry eye therapy, although this group was not specifically studied here.

Dry eye may be clinically very similar to mild cases of Meige's syndrome. ${ }^{7-10}$ Both diseases can adversely affect and be exacerbated by such activities as watching television, driving, and reading. Environmental conditions including bright light, wind, and air pollution are other common precipitants. Closing the eyes or relaxing in a dark, quiet room can alleviate the symptoms of both diseases. The relief that some Meige's syndrome patients experience with topical anaesthesia also supports the association between the syndrome and signals from the ocular surface.

Both dry eye and Meige's syndrome are strongly associated with abnormal blinking. ${ }^{9}$ It has been reported that the blink rate is normally up to 21 per minute, but may exceed 40 per minute in dry eye. ${ }^{13}{ }^{14}$ In mild Meige's syndrome or blepharospasm, increased blinking may be the only symptom. ${ }^{9}$ Whereas local ocular surface factors associated with dry eye may precipitate Meige's syndrome, once the condition becomes florid it may require treatment of more than the dry eye component for complete amelioration.

While it is important to note the association between dry eye and Meige's syndrome, it is essential to appreciate that they may also be distinctly separate entities. Patients with Meige's syndrome, but without any dry eye component may be misdiagnosed and improperly treated on the basis of just clinical symptoms, and conversely, Meige's syndrome patients with dry eye findings may not realise adequate relief unless the ocular condition is simultaneously treated. We consistently used established and objective criteria to diagnose dry eye. Similarly, we had the services of a neuropsychiatrist who employed video tape analysis to detect even subtle cases of Meige's syndrome.

We initially expected that many of the treatment unresponsive patients had Sjogren's syndrome because they had no reflex tearing and the severe ocular surface condition was typical of the disease. ${ }^{21}{ }^{27}$ It was thus surprising that only one patient with Meige's syndrome was also diagnosed with Sjögren's syndrome. The ocular surface may have been compromised by the Sjögren's syndrome so that topical treatment was only partially effective. The treatment unresponsive patients without Meige's syndrome had a variety of conditions; one had hemifacial spasm, one facial spasm, and two depression. However, the rest of the patients in this group did not have any definite ophthalmic or neuronal diagnosis, and further study is necessary to elucidate the cause of their severe symptoms, such as dermatochalasis. ${ }^{28}$
In conclusion, we have described an association between dry eye and Meige's syndrome. Although the pathogenesis of neither condition is established yet, both share certain common symptoms. When dry eye patients fail to respond to topical treatment, other diagnoses, including Meige's syndrome, should be considered.

This study was supported by the Japanese Ministry of Health and Welfare.

1 Toda I, Fujishima H, Tsubota $\mathrm{K}$. Ocular fatigue is the major symptom of dry eye. Acta Ophthalmol 1993;71:18-23.

2 Tsubota K, Toda I, Yagi Y, Ogawa Y, Ono M, Yoshino K. Three different types of dry eye syndrome. Cornea 1994;13:202-9.

3 Pflugfelder S, Huang A, Feuer W, Chuchovski P, Pereira I, Tseng S. Conjunctival cytologic features of primary Sjögren's syndrome. Ophthalmology 1990;97:985-93.

4 Tsubota K. New approaches to dry-eye therapy. Int Ophthalmol Clin 1994;34:115-28.

5 Tsubota K, Yamada M, Urayama K. Spectacle side panels and moist inserts for the treatment of dry-eye patients. Cornea 1994;13:197-201.

6 Hamano T, Ohashi Y, Cho Y. A new punctum plug. Am f Ophthalmol 1985;100:619-20.

7 Jordan D, Patrinely J, Anderson R, Thiese S. Essential blepharospasm and related dystonias. Surv Ophthalmol 1989;34:123-32.

8 Meige $\mathrm{H}$. Les convulsions de la face, une forme clinique de convulsion faciale, bilaterale et mediane. Rev Neurol (Paris) 1910;10:437-43.

9 Jankovic J. Etiology and differential diagnosis of blepharospasm and oromandibular dystonia. Adv Neurol 1988;49: 103-16.

10 Holds J, White G, Thiese S, Anderson R. Facial dystonia, essential blepharospasm and hemifacial spasm. Am Fam Phys 1991;43:2113-26.

11 Tolosa E, Marti M.J. Blepharospasm-oromandibular dystonia syndrome (Meige's syndrome): clinical aspects. In: Jankovic J, Tolosa E, eds. Advances in neurology. Vol 49: Facial dyskinesias. New York: Raven Press, 1988:73-84.

12 Marsden C. Blepharospasm-oromandibular dystonia syndrome (Brueghel's syndrome). A variant of adult-onset torsion dystonia? F Neurol Neurosurg Psychiatry 1976;39:12049 .

13 Tsubota K, Nakamori K. Dry eyes and video display terminals. N Engl f Med 1993;328:584

14 Tsubota K, Nakamori K. Effects of ocular surface area and blink rate on tear dynamics. Arch Ophthalmol 1995;113: 155-8.

15 Shimazaki J, and Dry Eye Research Group in Japan. Definition and criteria of dry eye. Ganka 1995;37:765-70.

16 Lemp M. Report of the National Eye Institute/Industry workshop on Clinical Trials in Dry Eyes. CLAO F 1995;21: 221-32

17 Norn M. Diagnosis of dry eye. In: Lemp M, ed. The dry eye. Heidelberg: Springer-Verlag 1992:133-82.

18 Toda I, Shimazaki J, Tsubota K. Dry eye with only decreased tear break-up time is sometimes associated with allergic conjunctivitis. Ophthalmology 1995;102:302-9.

19 Xu K, Yagi Y, Toda I, Tsubota K. Tear function index: a new measure of dry eye. Arch Ophthalmol 1995;113:84-8.

20 Schirmer O. Studien zur physiologie und pathologie der tranenabsonderung und tranenabfuhr. Albrech von Graefes Archiv fur Ophthalmologie 1903;56:197-291.

21 Tsubota K. The importance of the Schirmer test with nasal stimulation (letter). Am F Ophthalmol 1991;111:106-8.

22 Tsubota K, Xu K, Fujihara T, Katagiri S, Takeuchi T. Decreased reflex tearing is associated with lymphocytic infiltration in lacrimal glands. F Rheumatol 1996;23:31320.

23 Toda I, Tsubota K. Practical double vital staining for ocular surface evaluation. Cornea 1993;12:366-7.

24 Bisterveld O, Holland U. Diagnostic tests in sicca syndrome. Arch Ophthalmol 1969;82:10-4.

25 Udell IJ, Kenyon KR, Sawa M, Dohlman CH. Treatment of superior limbic keratoconjunctivitis by thermocauterization of the superior bulbar conjunctiva. Ophthalmology 1986;93:162-6.

26 Fox R, Robinson C, Curd J, Kozin F, Howell F. Sjogren's syndrome: proposed criteria for classification. Arthritis Rheum 1986;29:577-83.

27 Pflugfelder S, Huang A, Feuer W, Chuchovski P, Pereira I, Tseng S. Conjunctival cytologic features of primary Sjogren's syndrome. Ophthalmology 1990;97:985-93.

28 Vold S, Carroll R, Nelson J. Dermatochalasis and dry eye. Am f Ophthalmol 1993;115:216-20. 